\title{
Towards Understanding Consumers' Quality Evaluation of Online Health Information: A Case Study
}

Zequn Ye

University of Texas at Austin, USA. zequn@utexas.edu

Jacek Gwizdka

University of Texas at Austin, USA. asist2017@gwizdka.com

Yan Zhang

University of Texas at Austin, USA.

yanz@ischool.utexas.edu
Yalin Sun

University of Texas at Austin, USA. clairesun05@utexas.edu

Carla Teixeira Lopes

Universidade do Porto, Portugal. ctl@fe.up.pt

\begin{abstract}
We present a case study of quality evaluation of online health information. Two participants were selected from a health information search (HIS) study, in which we are investigating consumers' evaluation of the quality of online health information. The selected cases offered a rare example of two almost exactly opposite eye-movement patterns on the same webpage. To better understand the differences in these patterns, we investigated participants' cognitive evaluation processes by examining their textual explanations collected in post-task questionnaires and verbal explanations collected in the retrospective think-aloud (RTA) sessions. We discuss how eHealth literacy and personality scores may be related to the behavioral differences. The findings of this case study inform the formulation of hypotheses for full data analysis of the HIS study, as well as future research addressing behavior patterns and factors affecting consumers' quality evaluation of online health information.
\end{abstract}

\section{KEYWORDS}

eye-movement, quality evaluation, health information, eHealth literacy, personality

\section{INTRODUCTION}

The internet has become the most used, and often the first, go-to information source for ordinary health consumers (Marrie et al., 2013). The quality of online health information presents as a major concern for all stakeholders involved in the online health information ecosystem. Evaluating information quality remains to a major challenge for consumers (Feufel \& Stahl, 2012). Much research has been done to examine this topic. It was found that individual factors, such as age, education levels and health literacy, influence health information evaluation behavior (Lam \& Lam, 2012; Liao \& $\mathrm{Fu}, 2014)$. However, little is known about how consumers evaluate online health information quality and what exactly the challenges are.

$80^{\text {th }}$ Annual Meeting of the Association for Information Science \& Technology, Washington, DC | Oct. 27-Nov. 1, 2017

Authors Retain Copyright
In the online environment, hundreds of interface and content elements (e.g., author, dates and copyright) could serve as quality indicators (Zhang, Sun \& Xie, 2015). To enhance the current understanding of consumers' quality evaluation behavior, we adopted a mixed method approach, using eyetracking and retrospective interviews to examine what interface elements are being used for quality evaluation and whether individual differences, primarily eHealth literacy and personality, influence the behavior.

\section{METHODS}

We recruited 12 participants (we plan to recruit 48 in total). The study was conducted in a lab with a PC and an eye-tracking device. Participants first filled out demographic, personality and e-health literacy questionnaires. They then performed a practice task to ensure that everyone understands the study process. Then, each participant was presented with five predefined health information search tasks (orders were randomized). For each task, we preselected three webpages. Participants were asked to examine the three pages and determine whether they would recommend the pages to their family or friends. A retrospective think-aloud (RTA) session was performed after the completion of all the five tasks.

In this paper, we present data from two participants, because of their drastically different eye-movement patterns on one webpage. They were scanning and reading the page for the following scenario:

Imagine that one of your friends is struggling with whether to have her teenage son receive an influenza vaccine. So you conducted an online search, these three webpages are among the pages that show up in the search results. After browsing each page, you will determine whether you want to share this page to your friend. 


\section{RESULTS}

The gaze plots of the two participants show almost exactly opposite (i.e., not overlapping) eye-movement patterns (Figure 1). Gaze plots display the position, order and (roughly) time spent fixating at locations on a stimulus. P1's gaze plots roughly resemble an $F$, which corresponds to the F-shaped pattern in viewing text-based web content (Nielsen, 2006), and most of the fixations are on the main content area, where their sequence indicates focused reading of topical information (Gwizdka, 2014). By contrast, P2's gaze plots show an atypical pattern with fixations scattered at the top, right side and bottom areas, and only a few of them located in the main content area.

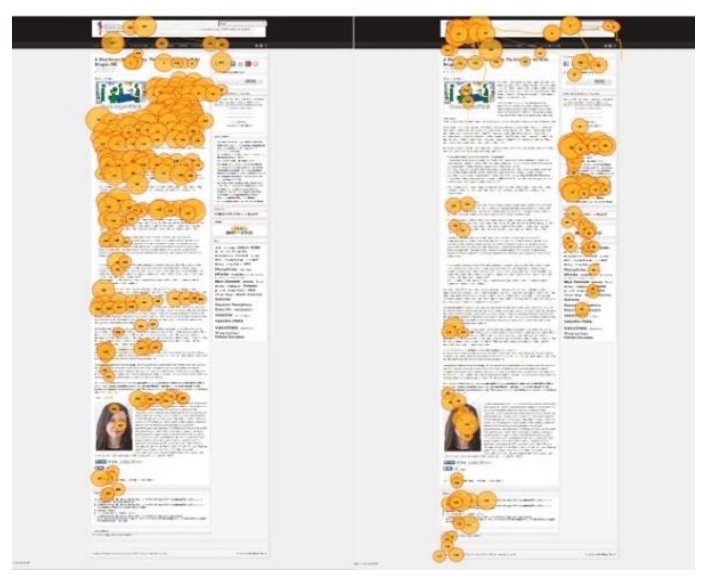

Figure 1. Gaze plots of $P 1$ (left) and $P 2$ (right)

Task performance of the two participants (including choice made on this page, time on task, time on page, count of links clicked on) and their background information (including eHEALS and TIPI scores) are shown in Table 1. P1's eHEALS score is substantially lower than P2. P1 spent significantly longer time (both time on task and time on page) than $\mathrm{P} 2$.

\begin{tabular}{|c|c|c|c|}
\hline & P1 & P2 \\
\hline \multicolumn{2}{|c|}{ Choice (share or not) } & No & No \\
\hline Time on Task & $176 \mathrm{~s}$ & $41 \mathrm{~s}$ \\
\hline \multicolumn{1}{|c|}{ Time on Page } & $85 \mathrm{~s}$ & $38 \mathrm{~s}$ \\
\hline \multirow{4}{*}{ Click on links } & To homepage & N/A \\
\hline \multirow{4}{*}{ TIPI } & eHEALS Score & 21 & 36 \\
\cline { 2 - 4 } & Extraversion & 1.0 & 5.5 \\
\cline { 2 - 4 } & Agreeableness & 4.0 & 6.5 \\
\cline { 2 - 4 } & Conscientiousness & 2.5 & 7.0 \\
\cline { 2 - 4 } & Emotional Stability & 4.5 & 3.5 \\
\cline { 2 - 4 } & Openness to Experiences & 6.0 & 7.0 \\
\hline
\end{tabular}

Table 1. Task performances, eHEALS and TIPI scores

Table 2 shows examples of web elements mentioned by the two participants in the RTA interviews and their short comment on these elements.

\section{SUMMARY}

The differences in eye-movement patterns can be attributed to many factors, such as one's demographic factors, familiarity with and interest in the task topic, as well as health literacy. Nevertheless, the preliminary observations based on currently collected data allow us to formulate working hypotheses. Here are a few plausible examples:

\begin{tabular}{|l|l|l|}
\hline & Page Element & Comment \\
\hline P1 & A cartoon & Not serious \\
\cline { 2 - 3 } & Social media widget & Not relevant \\
\hline \multirow{4}{*}{ P2 } & Title and logo image & Don't know what it is \\
\cline { 2 - 3 } & Layout and design & Doubting a lot \\
\cline { 2 - 3 } & Related posts & Very fear based \\
\cline { 2 - 3 } & Donate sign & A big no \\
\hline
\end{tabular}

Table 2. Example of phrases from RTA interviews

Compared with higher eHealth literacy, people with lower eHealth literacy tend to:

H1a: spend more time on quality evaluation;

H1b: rely more on relevance than on quality indicators.

In evaluating health-related webpage quality:

H2a: People with lower eHealth literacy tend to rely more on the main content of a webpage than on quality indicators;

H2b: People with higher eHealth literacy are able to take advantage of quality indicators.

We will begin testing these hypotheses after collecting data from more participants.

\section{REFERENCES}

Feufel MA. \& Stahl SF. (2012). What do web-use skill differences imply for online health information searches? J Med Internet Res, 14(3): e87.

Gwizdka, J. (2014). Characterizing relevance with eye-tracking measures. Proceedings of IIiX'2014 (pp. 58-67). New York, NY, USA: ACM.

Nielsen, J. (2006). F-shaped pattern for reading web content. Retrieved May 25th, 2017 from https://www.nngroup.com/articles/f-shaped-pattern-reading-web-content/

Lam, M. K., \& Lam, L. T. (2012). Health information-seeking behavior on the internet and health literacy among older Australians. Electronic Journal of Health Informatics, 7(2), 15.

Liao, Q. V., \& Fu, W. T. (2014). Age differences in credibility judgments of online health information. ACM ToCHI, 21(1), 2:1-2:23.

Marrie, R. A., Salter, A. R., Tyry, T., Fox, R. J. \& Cutter, G. R. (2013). Preferred sources of health information in persons with multiple sclerosis: Degree of trust and information sought. Journal of Medical Internet Research, 15(4), e67. http://doi.org/10.2196/jmir.2466

Zhang, Y., Sun, Y. \& Xie, B. (2015), Quality of health information for consumers on the web: A systematic review of indicators, criteria, tools, and evaluation results. JASIST, 66: 2071-2084 